\title{
OCTREOTIDE EN QUILOTÓRAX SECUNDARIO A CORRECCIÓN DE HERNIA DIAFRAGMÁTICA CONGÉNITA
}

John Camacho MD*, Marisol Duque MD**, Diana Arias MD***

\section{Resumen}

La presencia de quilo en la cavidad pleural es una situación rara cuya causa puede ser adquirida (trauma quirúrgico) o congénita (anomalías del sistema linfático), con manifestaciones clínicas variables dependientes de la cantidad y pérdida de quilo (desnutrición, alteración hidroelectrolítica e inmunosupresión). Se presenta una recién nacida con diagnóstico prenatal de hernia diafragmática izquierda con quilotórax de etiología traumática, a quien se realiza tratamiento médico mediante soporte nutricional y octreotide, con resolución y sin necesidad de optar por manejo quirúrgico.

\section{OCTREOTIDE FOR TREATMENT OF CHYLOTHORAX AFTER REPAIR OF CONGENITAL DIAPHRAGMATIC HERNIA}

\begin{abstract}
Chyle effusion in the pleural cavity is a rare situation. It may be acquired (surgical injuries) or congenital (abnormalities of the lymphatic system). Clinical manifestations are varied and depend on the amount of chyle loss (malnutrition, fluid and electrolyte imbalance and immune suppression). This article reports the case of a female newborn infant diagnosed with left diaphragmatic hernia who presented postoperative chylothorax. Medical management included nutritional support and octreotide. Resolution of chylothorax was obtained with no additional surgical treatment.
\end{abstract}

\section{Introducción}

El quilotórax es infrecuente ${ }^{1-4}$ y puede ser congénito o adquirido. ${ }^{1-1 \bullet}$ Se diagnostica por el análisis de sus características bioquímicas: células $>1.000 / \mu l$ con predominio de linfocitos $>80 \%$, triglicéridos $>100-110$ $\mathrm{mg} / \mathrm{dl}(1,1 \mathrm{mmol} / \mathrm{l})$ y proteínas $>20-30 \mathrm{~g} / \mathrm{dl}^{2,4,7,9-14} \mathrm{El}$ manejo se basa en medidas encaminadas a disminuir el flujo de linfa mediante dieta con triglicéridos de cadena media, nutrición parenteral total y hace poco con el uso de octreotide. ${ }^{1-6,9-12}$ Cuando dichas medidas fracasan se opta por procedimientos quirúrgicos..$^{1,4-6}$
Fecha recibido: octubre 8 de 2012 - Fecha aceptado: noviembre 22 de 2012

* Residente II de Pediatria, Fundación Universitaria de Ciencias de la Salud. Bogotá DC, Colombia.

** Residente III de Pediatría, Fundación Universitaria de Ciencias de la Salud. Bogotá DC, Colombia.
*** Médica pediatra, neonatóloga. Jefe del posgrado de pediatría. Instructor Asistente, Fundación Universitaria de Ciencias de la Salud. Bogotá DC, Colombia. 


\section{Presentación del caso}

Recién nacida a término, de 37 semanas por Ballard, con peso adecuado para la edad gestacional, llevada a cesárea por presentación podálica, con diagnóstico prenatal de hernia diafragmática izquierda. Nace no vigorosa con Apgar 3, 6 y 8, requiere reanimación neonatal e intubación orotraqueal, además se evidencia arteria umbilical única y ano imperforado, los gases del cordón son compatibles con depresión neonatal leve, por lo cual se traslada a la unidad de recién nacidos. Durante la evolución se inicia nutrición parenteral total, se lleva a cirugía a los dos días de vida para realizar herniorrafia diafragmática izquierda, toracostomía izquierda y colostomía derivativa a nivel de recto sigmoide sin complicaciones. Ante la sospecha de sepsis neonatal temprana se inicia ampicilina y amikacina. Se coloca en fototerapia por hiperbilirrubinemia; estando en ventilación mecánica revela parámetros ventilatorios altos por lo cual se solicita ecocardiograma que muestra ductus arterioso permeable de 1,8 $\mathrm{mm}$ sin repercusión hemodinámica e hipertensión pulmonar leve. Se inicia transfusión de glóbulos rojos empaquetados y plaquetas. Maneja parámetros ventilatorios bajos que permiten la extubación y retiro de la toracostomía al décimo día de vida. Paciente con sospecha de asociación VACTERL por la hernia diafragmática izquierda asociada con ano imperforado, ductus arterioso persistente y arteria umbilical única. Se descartan malformaciones renales, vertebrales y fístulas traqueoesofágicas. Al día 18 de vida presenta pico febril con disminución marcada del murmullo vesicular en hemitorax izquierdo y aumento de requerimientos de oxígeno. La radiografía de tórax evidencia líquido en cavidad pleural izquierda con desviación del cardiomediastino (Figura 1) y se decide iniciar cefepime y vancomicina previa toma de policultivos.

Se realiza toracentesis guiada por ecografía de tórax que muestra liquido amarillo turbio que presenta las siguientes características: LDH 235 U/l, proteínas $2,4 \mathrm{~g} / \mathrm{dl}$, leucocitos 4.400 , neutrófilos $7 \%$, linfocitos $93 \%$, criterio de light compatible con exudado (proteínas de líquido pleural/proteínas de sangre $>0,5$ ) y triglicéridos $181 \mathrm{mg} / \mathrm{dl}$. Se considera quilotórax

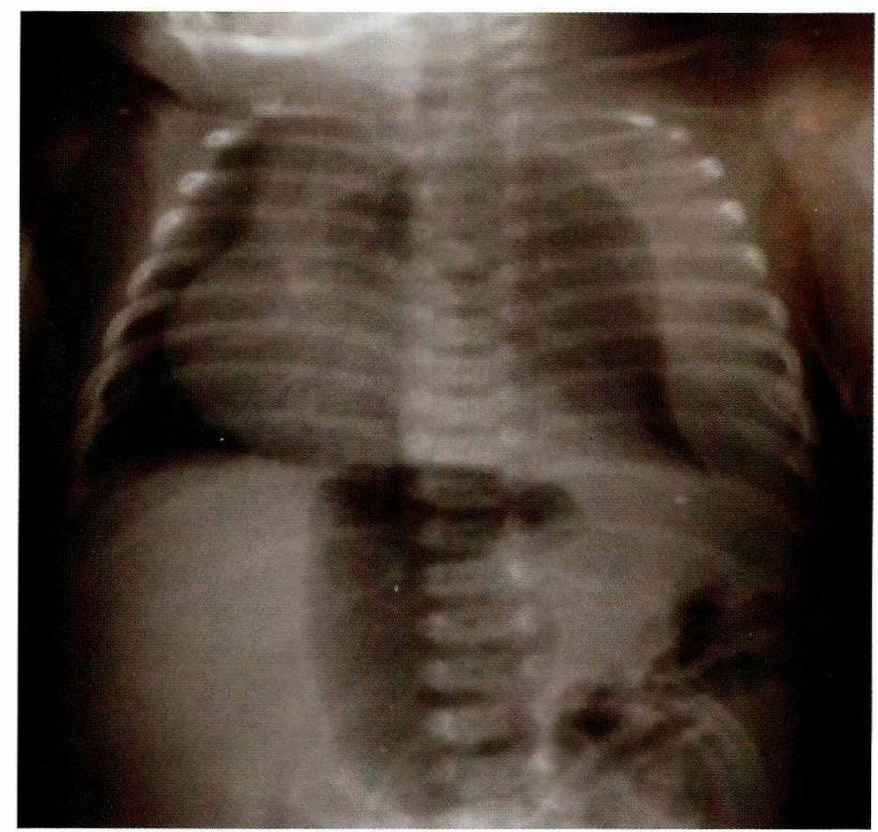

Figura I: Radiografía de tórax con el líquido en la cavidad pleural izquierda.

postraumático, al vigésimo día de vida se inicia nutrición parenteral sin lípidos y manejo nutricional enteral mínimo con triglicéridos de cadena media libre de gluten y sacarosa. La evolución es adecuada sin signos de dificultad respiratoria, requiere oxígeno suplementario con bajo flujo, se aísla en el hemocultivo Klebsiella pneumoniae sensible a cefepime, sin aumentar el trabajo respiratorio.

La radiografía de control a las 48 horas reveló importante aumento de la colección a pesar del manejo nutricional instaurado, por lo cual al vigésimo sexto día de vida se decide iniciar octreotide a $1 \mu \mathrm{g} / \mathrm{k} / \mathrm{hora}$, se realiza descenso progresivo de la nutrición parenteral y enteral completa. Van diez días de antibioticoterapia. Dado que el paciente no presenta deterioro clínico, con patrón respiratorio adecuado y sin aumento de los requerimientos de oxígeno, se continúa el manejo médico. El nuevo control radiológico al quinto día no muestra aumento de la colección pleural, se procede a aumentar el octreotide a $3 \mu \mathrm{g} / \mathrm{k} / \mathrm{hora}$ al trigésimo día de vida, suspendiendo la nutrición parenteral total en forma progresiva y continuando aporte oral completo con fórmula de triglicéridos de cadena media. 
La evolución es favorable sin signos de dificultad respiratoria ni de respuesta inflamatoria sistémica, completa doce días de infusión con octreotide observando resolución del quilotórax, indicación para suspenderlo el trigésimo tercer día de vida. El control radiológico revela cavidades pleurales libres, el paciente no muestra recidiva del derrame, hay ganancia de peso adecuada y tolera la nutrición enteral especial completa. Durante el tratamiento no se reportaron reacciones adversas secundarias al uso de octreotide. Egresó sin complicaciones y la buena respuesta al octreotide evitó procedimientos invasivos y sus complicaciones.

\section{Discusión}

El quilotórax se define como la presencia de fluido linfático o quiloso en la cavidad pleural ${ }^{1,2,3}$, es una situación infrecuente con predominio en hombres $(2: 1){ }^{2}$ La incidencia neonatal es de 1:1.000 a 1:15.000 gestaciones $^{4}$ y es de 10 a $30 \%$ después de procedimientos quirúrgicos cardiotorácicos en especial la corrección de hernia diafragmática congénita ${ }^{5,6}$ con predominio del lado izquierdo. El flujo de quilo en condiciones basales es de $1,38 \mathrm{ml} / \mathrm{k} / \mathrm{h}$ y en neonatos puede llegar a $250 \mathrm{ml} /$ día. Algunas condiciones como alimentación abundante en lípidos aumenta hasta diez veces el valor basal. ${ }^{1,8,9}$ La principal característica del quilo es su elevada concentración de lípidos, linfocitos y proteínas. ${ }^{14}$ La etiología exacta se desconoce pero algunas teorías sugieren: 1) alteración en el origen como la variación de los vasos linfáticos asociados con hernia diafragmática; 2) aumento de la presión del conducto torácico que lleva a rupturas del mismo; y 3) daño directo de los vasos linfáticos (postraumático) que conlleva escape de quilo a la cavidad pleural. ${ }^{5,6,10}$ Se clasifica como congénito ò no traumático cuando hay malformaciones del conducto torácico, agenesia o atresias ${ }^{10,12}$; puede asociarse con malformaciones de otros sistemas, linfangiectasias, enfermedades cardíacas congénitas, malignidades mediastinales y cromosomopatías. ${ }^{1,8}$ Es adquiridoó traumático como complicación intraoperatoria de cirugías cardiotorácicas que lesionan el conducto torácico ${ }^{1,3,7,10}$, como sucede después de correcciones cardíacas, pulmonares, esofágicas y diafragmáticas
7,9,10.12.13 debido a las múltiples variaciones anatómicas de dicho conducto. ${ }^{4.813}$ En algunos casos de trombosis $u$ obstrucción de la vena cava superior ocurre un aumento retrógrado de presión hacia los vasos linfáticos. ${ }^{12} \mathrm{La}$ severidad de las manifestaciones clínicas depende de la cantidad de quilo alojado en la cavidad pleural ${ }^{3}$, que puede condicionar grados de dificultad respiratoria variables hasta la insuficiencia, además de bajos puntajes de Apgar que requieren reanimación, cuidado intensivo y ventilación mecánica. ${ }^{9}$ En casos congénitos cuando la cantidad de quilo es importante, puede asociarse con hipoplasia pulmonar. ${ }^{1,3}$

El quilotórax adquirido se manifiesta en la clínica por signos de dificultad respiratoria, limitación para la expansión pulmonar, semiología de derrame pleural y disminución del murmullo vesicular, en un paciente con el antecedente quirúrgico. ${ }^{9}$ Se desarrolla entre el primer día del trauma hasta el décimo octavo posoperatorio. ${ }^{7}$ Entre las complicaciones derivadas de la pérdida de quilo se encuentran desnutrición y edema, alteración hidroelectrolítica, inmunosupresión y aumento de la predisposición a infecciones (secundaria a pérdida de linfocitos e inmunoglobulinas). ${ }^{1,6,9,10} \mathrm{El}$ diagnóstico prenatal se puede establecer por ecografía obstétrica. ${ }^{9}$ En el período neonatal además de los antecedentes y la clínica, la radiografía del tórax constituye la herramienta inicial en la cual se observa borramiento de los senos costofrénicos o desplazamiento del mediastino. ${ }^{10,14}$ La ecografía pleural cuantifica el volumen con la ventaja de no irradiar al paciente y permitir ubicar el sitio de punción..$^{9,14} \mathrm{El}$ análisis suele revelar un aspecto lechoso, $\mathrm{pH}$ alcalino, células $>1.000 / \mu \mathrm{l}$ con predominio linfocitario $>80 \%$ (importante porque su valor no varía con la dieta), triglicéridos $>100-110$ $\mathrm{mg} / \mathrm{dl}(1,1 \mathrm{mmol} / \mathrm{l})$, proteínas $>20-30 \mathrm{~g} / \mathrm{dl}$. $^{2,4,7,9-11,13,14}$ El manejo tiene varias opciones médicas y quirúrgicas. $\mathrm{Al}$ comienzo el de tipo conservador puede ser efectivo en cerca del $80 \%$ de los pacientes. ${ }^{4,6}$ Incluye el soporte nutricional donde se elimina la dieta grasa por medio de fórmulas lácteas especiales ricas en triglicéridos de cadena media ${ }^{1,4,6}$, estas moléculas con menos de doce carbonos se absorben vía portal lo cual reduce el flujo de linfa disminuyendo la cantidad del derrame., ${ }^{9,10,15}$ También se utiliza la nutrición parenteral total sin ácidos grasos ${ }^{1,2,5,12}$, con los riesgos derivados del uso 
a largo plazo como son la colestasis, infección y trombosis. " Aunque no hay protocolos, la mayoría de los autores recomiendan el drenaje del líquido pleural por toracentesis y en caso de recidiva mediante toracostomía, con bastante variabilidad sobre la duración de esta medida, el tiempo de resolución $n^{4.569}$ y la posibilidad de complicaciones asociadas con dicho procedimiento. González R. y colaboradores encontraron un promedio de duración de 27 días con tubo de toracostomía. ${ }^{5}$

En los casos refractarios al tratamiento médico se opta por el manejo quirúrgico aunque no se ha precisado el momento en el cual el paciente debe ser llevado a cirugía. Algunos autores lo recomiendan cuando hay persistencia del quilotórax por más de dos a cuatro semanas, si la producción de quilo es $>100 \mathrm{ml} / \mathrm{año}$ de edad niño/día o bien $>10-15 \mathrm{ml} / \mathrm{kdía}$ por cinco días ó la aparición de complicaciones nutricionales. ${ }^{5,7,10,11}$ Entre las medidas quirúrgicas están la toracotomía abierta para ligar el conducto torácico, cortos circuitos pleuroperitoneales y pleurodesis entre otras. ${ }^{1,4,5,5,7,10}$

Algunos reportes muestran la efectividad del octreotide (análogo sintético de la somatostatina con una vida media más prolongada) para el manejo del quilotórax..$^{1-6,9-11}$ No se ha dilucidado con exactitud su mecanismo de acción, pero se cree que reduce la producción de quilo debido a múltiples efectos inhibitorios a nivel central (hormona de crecimiento y tirotropina) y del tracto gastrointestinal, actuando en los receptores de somatostatina en la circulación esplácnica (disminuye la secreción de secretina, péptido intestinal vasoactivo, colecistocinina y serotonina entre otros). En forma secundaria reduce la motilidad gastrointestinal, el flujo esplácnico por vasoconstricción arteriolar y la absorción intestinal de lípidos. ${ }^{3,5,9,11} \mathrm{La}$ dosis intravenosa varía entre 0,3 y $40 \mu \mathrm{g} / \mathrm{k} / \mathrm{hora}$ y se incrementa según el volumen del quilotórax. Tiene una vida media de una a dos horas, el pico de acción es entre 24 y 48 horas que es cuando hay más evidencia de disminución del quilotórax y la duración del tratamiento depende de la baja producción de quilo. $2,3,4,11$ Entre las reacciones adversas se encuentran emesis, náuseas, hiper o hipoglicemia, elevación de enzimas hepáticas, esteatorrea y trastornos tiroideos. ${ }^{3,10}$

\section{Referencias}

1. Rasiah SV, Oei j, Lui k.Octreotide in the treatment of congenital chylothorax. J Paediatr Child Health. 2004 Sep-Oct;40(9-10):585-8

2. Altuncu E, Akman I, Kiyan G, Ersu R, Yurdakul Z, Bilgen H, Ozdoḡan T, Ozek E.Report of three cases: congenital chylothorax and treatment modalities. Turk J Pediatr. 2007 Oct-Dec;49(4):418-21.

3. Kalomenidis I. Octreotide and chylothorax. CurrOpinPulm Med. 2006 Jul;12(4):264-7.

4. Paget-Brown A, Kattwinkel J, Rodgers BM, Michalsky MP. The use of octreotide to treat congenital chylothorax. J Pediatr Surg. 2006 Apr;4l(4):845-7.

5. Gonzalez R, Bryner BS, Teitelbaum DH, Hirschl RB, Drongowski RA Mychaliska GB. Chylothoraxafter congenital diaphragmatic hernia repair. J Pediatr Surg. 2009 Jun;44(6):1181-5.

6. Goyal A, Smith NP, Jesudason EC, Kerr S, Losty PD. Octreotidefor treatment of chylothorax after repair of congenital diaphragmatic hernia.J Pediatr Surg. 2003 Aug;38(8):E19-20.

7. Büttiker V, Fanconi S, Burger R. Chylothoraxin children:guidelinesfordiagnosis and management. Chest.1999Sep;116(3):682-7.

8. Wegner A,Wegner ME, Milad M. Quilotórax en el período neonatal: caso clínico y revisión de la literatura. Rev. chil. pediatr. 1999 Nov; 70(6): 498-504.

9. CrehuáGaudiza E, Izquierdo Macián I, Fernández Gilino C, Gutiérrez Laso A, Morcillo Sopena F, Vento Torres M. Quilotórax neonatal congénito y posquirúrgico revisión y algoritmo diagnóstico-terapeútico. Acta Pediatr Esp. 2006; 64: 276-282.

10. Benítez I, Copons C, Castillo F. Tratamiento del Quilotórax. AnPediatrContin. 2008;6(3):159-165.

11. CoponsC, Benítez I., Castillo F. Salcedo S. Quilotórax neonatal: etiología, evolución y respuesta al tratamiento.AnPediatr (Barc). 2008;68(3):224-31.

12. Densupsoontom NS, Jirapinyo P, Wongam R, Thamonsiri N, Nana A, Laohaprasitiporn D, et al. Management of chylothorax and chylopericardium in pediatric patients: experiences at Siriraj Hospital, Bangkok.Asia Pac J ClinNutr. $2005 ; 14(2): 182-7$.

13. Milonakis M, Chatzis A, Giannopoulos N, Contrafouris C, Bobos D, Kirvassilis G, et al. Etiology and management of chylothoraxfollowing Pediatric Heart Surgery. J Card Surg. 2009;24:369-373.

14. Efrati O, Barak A. Pleural effusions in the pediatric population. Pediatr Rev. 2002 Dec;23(12):417-26.

15. Gershanik J, Jonsson H, Riopel D, Packer R. Dietary Management of Neonatal Chylothorax. Pediatrics. 1974;53;400-403. 